\title{
Examining the Mental Health Issues Prevalent in Adolescent Type-1 Diabetics: An Evidence-Based Review to Improve Health
}

\section{Kristen Goad*}

Graduate Student, Graduate Student, MCPHS University, Longwood Ave, Boston, MA, USA

*Corresponding author: Kristen Goad, Graduate Student, MCPHS University, Longwood Ave, Boston, MA, USA, Tel: 603-991-9334; E-mail: kgoad23@gmail.com Rec Date: March 31, 2015; Acc Date: August 27, 2015; Pub Date: August 31, 2015

Copyright: (c) 2015 Goad K. This is an open-access article distributed under the terms of the Creative Commons Attribution License, which permits unrestricted use, distribution, and reproduction in any medium, provided the original author and source are credited.

\begin{abstract}
The purpose of this paper is to explore the mental health comorbidities present in adolescent type-1 diabetics and the current process and treatment available for this illness. An integrative review of 10 articles discussing mental health problems present in diabetic patients was performed. Although not every article had the exact preferred population of adolescent type-1 diabetics they did address one or more issues of concern. Many of the studies reviewed found direct links between self-reported depression, anxiety or poor quality of life and increased glycosylated hemoglobin (A1c) values. The CINAHL and Medline databases was use to search articles, articles older than five years were excluded and key phrases used for searching were; type-1 diabetes, adolescents, depression, anxiety and eating disorders. Many of the studies only addressed how to evaluate for mental health disorders; there was not an abundance of follow-up with these studies. There were links found between depression and poor A1c value but only a few studies addressed interventions once a mental health disorder had been identified.
\end{abstract}

Keywords: Type-1 Diabetes; Adolescent; Eating disorders; Anxiety; Depression

\section{Introduction}

Type-1 diabetes is a chronic illness most often diagnosed from childhood to young adulthood [1]. When a new diagnosis is made there is a large focus on blood sugar levels, A1c management and preventing medical complications. What can easily be overlooked is the mental health of the patient. With any chronic illness diagnosis the risk of depression can increase, but with an adolescent population already experiencing many life changes this risk can be even greater. Depression is one mental health concern but the danger of eating disorders must also be examined. Eating disorders are not uncommon during adolescence but they can cause even greater danger to the adolescent with type-1 diabetes. Insulin omission and dose reduction are methods of weight reduction used by adolescents that can cause significant negative effects to glycemic control [2]. While there is a vast amount of research available on diabetes only about $5 \%$ of diabetics are type-1 [1]. Much of the reviewed research focused on medical control and nutrition, there was very little research found on mental health issues prevalent in any diabetics, specifically adolescent type-1 diabetics. Depression and eating disorders can cause serious health problems and affect a patients quality of life, and type- 1 diabetes can be difficult to manage, and poses many health risks. What is not known is how these interact and how to help identify and manage the mental health issues seen in this patient population [3].

It has been estimated that the risk for depression is nearly double in adolescents with type- 1 diabetes in comparison to adolescents without the illness [4]. A recent long-term follow up study found that $50 \%$ of type-1 diabetics were diagnosed with a mental illness within one year of the diabetes diagnosis, the most common illness being depression. Managing type-1 diabetes while experiencing anxiety can be particularly challenging and may lead to poor disease management [3].
Eating disorders are also a significant risk; one study reported that nearly $10 \%$ of participants had admitted to skipping insulin doses and purging to help lose weight, leading to increased difficulty with medication management [2]. Depression when associated with diabetes can lead to decreased self-care including poor glycemic control, increased risk of long-term complications, and decreased quality of life [4]. With the risks of mental health issues combined with type- 1 diabetes being so severe, the detection and treatment of mental health issues is of high importance. Programs should be in place to assist practitioners in providing mental health screening and making mental health resources available for adolescent type-1 diabetics. A primary care provider needs to care for all aspects of the patient and catching any signs or symptoms of depression, anxiety or eating disorders can make an impact on the long-term effect on glycemic control and quality of life [3]. The question guiding the integrative review is:

\section{Methodology}

An integrative review was conducted to examine the current literature on the concern of mental health disease in adolescent type- 1 diabetics. This method was chosen to explore current research, analyze evidence and identify the interventions to assist future family nurse practitioners to support adolescents with type-1 diabetes. There are five important steps in conducting an integrative review, the first is to identify the problem, once a problem is identified the literature search and collection of pertinent data is completed. Once sufficient data has been obtained it must be reviewed and evaluated, once evaluation is complete the data will be analyzed. After a thorough analysis the data can be interpreted and presented in a way that will tie everything together. A well-conducted integrative review will provide current and accurate representation of the evidence available on the chosen topic and will identify further research needed. 
The population under investigation includes adolescents between the ages of 10-19 and diagnosed with type-1 diabetes. Inclusion criteria involved selection of articles between 2004 and 2014 with a referenced population of type-1 diabetics, adolescents, and mental health disorders associated with diabetes and adolescent age. There were many articles on type-two diabetes and on young children (age $<10$ years) with type- 1 diabetes and families of young children with type- 1 diabetes but these were excluded, as they did not relate to the specified population of adolescents. Articles that addressed mental health issues in type-1 diabetics were still used even if the specific population was not adolescents. Journals and articles were also limited to those in English.

Information for the integrative review was obtained primarily from peer-reviewed scholarly journals, the American Diabetes Association and reviewing diabetes centers websites such as the Joslin Clinic in Boston, MA, the Hallett Center in Providence, RI, and The Diabetes Center of New England in Stoughton, MA. The websites were reviewed to identify and determine types of services advertised as being available and if mental health was listed on these websites.

Many of the articles utilized the SEARCH for diabetes in youth research group to review multiple qualitative studies. SEARCH is a national study that seeks to attain information from participants to learn and understand diabetes among children and young adults in the United States. The articles for review were obtained by utilizing a search on the CINAHL and Medline databases using the following keywords: eating disorder, depression, mental health, adolescence and type- 1 diabetes. Articles were then reviewed by relevancy to the concern of mental health issues in adolescent type-1 diabetics and date of publication. The studies were reviewed to ensure adequate sampling; if inadequate sampling was present this may lead to decreased validity by not providing an appropriate population sample, which may skew results. The demographics of the studies were also reviewed to determine if the collected studies and target population matched. Any studies with discrepancies, such as inaccurate testing or contradictory results were not used for the purpose of this review. The methodology of each study was reviewed to determine if the process was appropriate to provide valid findings by assessing sample selection and type of study/review conducted. The studies were reviewed without bias to avoid a threat to validity. Articles were reviewed by age of publication, reviewing older articles first to help identify the progress of research on the topic through the years. Each article was critiqued separately and the level of evidence was determined. Level of evidence is determined by assessing the quality of design, validity, and applicability to patient care [5].

\section{Critique of Research Studies}

Markowitz, Butler, Volkening, Antisdel, Anderson and Laffel published a cross-sectional study in 2010 validating a diabetes-specific screening tool for disordered eating. The aim of the study was to update and validate a current screening tool already in place, the Diabetes Eating Problem Survey (DEPS). The 28 item self-reporting survey was based on patient's own personal habits and higher scores indicated disordered eating habits. Markowitz et al. [6] utilized this DEPS survey on 112 participants and used the results to formulate a revised version that was more directed toward the adolescent age group and disordered eating habits. The new survey, the DEPS-R, resulted a 16-item survey with the same likert rating scale for each item ( $0=$ never-5=always). The revised survey was also administered to the 112 participants.
The study reported females scored significantly higher on the DEPS$\mathrm{R}$ study than males. Individuals with higher A1c values were also noted to be the same individuals who admitted to missing or restricting their insulin doses, these same participants also tended to score $>20$ on the DEPS-R ( $\mathrm{p}=.0001$ ). Participants with scores $>20$ and considered to be restricting insulin also tended to have lower BMI scores implying participants may be using insulin omission or restriction for weight loss purposes. "For individuals with type 1 diabetes, insulin restriction is a unique disordered eating behavior that is used to induce weight loss" [6].

According to the American Psychiatric Association, 2013, bulimia nervosa and binge eating disorder are more likely to have an onset during the late adolescent to early adulthood years. Although Markowitz et al., [6] utilized a relatively small sample size it demonstrated a positive correlation between measurable variables such as BMI $(\mathrm{p}=.01), \mathrm{Alc}(\mathrm{p}=.001)$ and the patient's own report of the diabetes self-care in regard to disordered eating. Utilizing a short screening tool for disordered eating in adolescents can help identify both at risk patients and patients that may already have an eating disorder.

McGrady and Hood [3] sought to determine the correlation between depressive symptoms, blood glucose monitoring and glycemic control among adolescents with type-1 diabetes. Using a longitudinal study over a period of six months, $144(\mathrm{n}=144)$ participants completed the 27 item Children's Depression Inventory (CDI) selfreporting scale (0-2), scores of 13 or higher were highly suggestive of depression requiring immediate attention. $25 \%$ of participants displayed clinical symptoms; negative mood, anhedonia and feelings of ineffectiveness. The more significant the feelings of ineffectiveness and negative mood, the less likely patients were to have effective blood glucose monitoring frequency both at the same time point and six month follow up $(\mathrm{p}<.05)$. Higher A1c levels were associated with more depressive symptoms as rated on the CDI at the same time point but none were related to A1c six months later $(\mathrm{p}>.05)$.

McGrady and Hood [4] identified areas needed for future study including the link between blood glucose monitoring, Alc levels and depressive symptoms. Although depressive symptoms were not clinically evaluated and based solely on self-reporting, significant links between poor diabetes self-management and depressive symptoms were observed. A lack of clinical evaluation in conjunction with selfreporting is a limitation. Any changes between the links were not reviewed whereby future studies may consider to predict long-term effects of depressive symptoms $(\mathrm{p}<.01)$.

Masdin and Klejdys [7] implemented a counseling service for their type- 1 diabetes patients to help address their anxiety related to life events. The counseling sessions lasted 50 minutes and occurred once weekly for six weeks. They used a retrospective study to review A1c values, and Clinical Outcomes in Routine Evaluation (CORE) questionnaire results to help determine the efficacy of the newly implemented counseling sessions. Although the sessions were not limited to adolescents they did address a significant topic of anxiety and it was narrowed down to type-1 diabetes patients.

At the end of six weeks, participants notably had significant lower levels of anxiety ( $p=.001$ ), as well as improved A1c values; $\mathrm{Md} 9.5 \%$ pre-counseling to Md 9.3\% post-counseling $(\mathrm{p}=.007)$. These results are solely based in one clinic that implemented counseling without large amounts of funding for the study. Although the Masdin et al., results 
are promising, additional research is required to determine the longterm outcomes of counseling and the effects of it on adolescents.

In Mental Health Issues in Adolescents and Young Adults with Type-1 Diabetes: Prevalence and Impact on Glycemic Control written by Bernstein et al. [2], a cross-sectional study of 150 patients $(n=150)$, three screening tools were used to determine mental health disorders and associated A1c values. Using a 21-item self-report Beck's Depression Inventory (BDI) determined level of depression, scores of $>14$ indicate depression, increasing scores correlate with severity of depression. The second of the three screening tools, the 41-item, selfreporting Screen for Child Anxiety Related Emotional Disorders-41 (SCARED-41) sought to determine adolescent anxiety whereby scores $>25$ indicate positive anxiety. The last screening tool used was the Eating Disorder Screen for Primary Care (ESP), this tool consists of five questions screening for disordered eating, answering yes to one or more questions indicates a positive screen.

One-third (34.7\%) of the participants scored positive on at least one of the screening tools, those who screened positive experienced twice the amount of change of poor diabetes control compared to those who did not screen positive. Higher Alc values correlated with younger age and also younger age at time of diagnosis had higher Alc values. A small percentage of participants admitted to taking less insulin than the directed dosage.

There was a direct correlation between poor diabetes control and positive screening test for depression $(\mathrm{p}=.67)$, anxiety $(\mathrm{p}=.09)$, and disordered eating $(\mathrm{p}=.13)$. Since adolescents are already at a vulnerable stage in life this study of adolescents revealed type-1 diabetic adolescents are at an increased risk for mental health comorbidity, which may lead to poor health outcomes. These results correlate with the findings from the 2010 article published by McGrady \& Hood showing increased depressive symptoms were related to decreased blood glucose monitoring and increased A1c values.

In 2014, Claude et al. [8] participants with type-1 or 2 diabetes for health anxiety. 414 participants $(n=414)$ completed the Health Anxiety Inventory-Short Form (SHAI), an 18-item questionnaire, scores greater than 20 are indicative of increased health anxiety. The StateTrait Anxiety Inventory (STAI) scale was also used, consisting of 20 items and scores ranging from 20-80, the higher the score the higher the anxiety level. The Fear of Complications Questionnaire (FCQ) scale was used to assess fear of complications such as loss of eyesight or kidney dysfunction. The 15 -item scale ranges from $0-45$, with the higher score the greater the fear. The World Health Organization Quality of Life was used as well; a 26-item survey to help determine self-perceived quality of life. The last scale used was the Summary of Diabetes Self-Care activities, which measures self-reported adherence to diabetes specific self-care activities.

One quarter of participants had positive scores for the SHAI questionnaire, however there were no differences noted between type-1 and 2 diabetics. Higher levels of anxiety were seen in younger ages ( $p=$. $001)$, single participants $(\mathrm{p}=.001)$, and a shorter length of diagnosis $(\mathrm{p}=.01)$. Higher levels of anxiety were correlated to decreased physical quality of life $(\mathrm{p}=.001)$. Although the population under investigation included both type- 1 and 2 diabetics and were not narrowed down to adolescents the results revealed important issues for diabetics. Whereby, there were no differences between type-1 or 2 diabetes but notably levels of anxiety among younger female individuals increased $(\mathrm{p}<.001)$. Although health anxiety was not correlated to blood glucose testing $(\mathrm{p}>.01)$ these findings are consistent with other literature suggesting an increase of mental illness exists among younger populations with diabetes. A 2012 study by Bernstein et al. [2] also reported type-1 diabetic adolescents (one-third of 150 participants) screening positive for depression, anxiety, or disordered eating.

In a randomized controlled trial consisting of 139 participants $(n=139)$ with type- 1 or 2 diabetes, the effects of a mindfulness-based cognitive therapy (MBCT) on their perceived quality of life, and glycemic control were evaluated. Participants with low scores $(<13)$ on the World Health Organization-5 Well Being Index were randomly assigned to a MBCT group or a control group. The MBCT group received eight weekly two-hour sessions in groups of 4-10 and the program focused on developing mindfulness using meditation [9]. Assessments were conducted prior to the sessions beginning, the middle of the sessions (4 weeks), and after the completion of the sessions ( 8 weeks). The control group was able to take part in the MBCT after the study had been completed to allow them the same counseling as the experiment group.

Significant improvements were noted in mental $(\mathrm{p}=.003)$ and physical ( $\mathrm{p}=.032$ ) quality of life in the MBCT group when compared to the control group. The MBCT group experienced lower perceived levels of stress $(\mathrm{p}=.001)$, symptoms of depression $(\mathrm{p}=.006)$ and anxiety $(p=.019)$. No significant changes were seen in Alc values for the MBCT group over time; yet, the control group however, exhibited higher Alc values over time. High dropout rate $(26 \%)$ and use of a non-active control were limitations to the 2013 Van Son et al study. Although both type- 1 and 2 diabetics were included in the Van Son et al., 2013 study and there were no age limitations, the results provide extremely useful insight to the use of mindfulness-based therapy as a means to assist patients to manage stress and anxiety.

In 2013 Powers, Richter, Ackard, Critchley, Meier and Criego reviewed two commonly used disordered eating questionnaires and evaluated their ability to predict disordered eating in type-1 diabetic patients [10]. The Eating Disorders Examination Questionnaire (EDEQ) is a 38-item self-report that assessed disordered eating habits over the previous 28 days. It addressed dietary restraint, eating concern, weight concern, and shape concern. The higher the score the more disordered eating habits. The second questionnaire assessed was the Eating Disorders Inventory version 3 (EDI-3). This is a 91-item selfreport that rates each question on a 6-point likert scale; higher scores imply higher levels of disordered eating habits. The scale addresses 12 scales including; drive for thinness, bulimia, body dissatisfaction, low self-esteem, personal alienation, interpersonal insecurity, interpersonal alienation, interoceptive deficits, emotional dysregulation, perfectionism, asceticism, and maturity fears.

An expert panel to evaluate the items in each questionnaire and determined if having type- 1 diabetes influenced the response to each item. Two groups completed the surveys. One group consisted of 48 patients with an eating disorder and type-1 diabetes; the second group was 96 patients with an eating disorder but no type-1 diabetes.

After review the experts rated half of the items on the EDE-Q to have a high level of influence for the type-1 diabetes participants. Frequency of overeating within the last four weeks had a high level of influence $(\mathrm{p}=.041)$. Dietary restraint questions also showed high level of influence. Avoiding certain foods $(\mathrm{p}=.012)$, going long periods without eating $(\mathrm{p}=.001)$ and setting definite rules for eating $(\mathrm{p}=.002)$ were the questions with highest levels of influence. As for the EDI-3 only 6 of the 91 items showed high levels of influence. Of the highest influence were questions that addressed bulimia symptoms $(p=.005)$ 
and drive for thinness $(\mathrm{p}=.019)$. Both groups had levels that indicated an eating disorder with clinical impairment. Though both groups scored positive, it was found that the type-1 diabetes group had less frequently unhealthy behaviors and tended to have less negative feelings toward eating. The results suggest that the type- 1 diabetes group have healthier relationships regarding food, weight, and shape than the non type-1 diabetes group. The results revealed the questions with the highest Type-1 diabetes influence were also the questions that could be more easily misinterpreted. Since the questionnaires are selfreport, patients may interpret each question differently and may over or underestimate their responses. The most important way of preventing misinterpretation is to discuss the answers with the patient to understand what exactly they meant when they answered the question. The authors advocate for further development of a type-1 diabetes specific questionnaire to address eating disorder habits. An expert review is a low level of evidence but provides excellent background to help build future research and randomized controlled trials utilizing the questionnaires would be beneficial.

In 2013, Herbert et al. and interviewed a sample of 13 type- 1 diabetes patient-parent duos regarding their experiences at the Teamwork Project [11]. The teamwork Project was a randomized controlled study (level II evidence) designed to help prevent poor glycemic control in adolescents with type-1 diabetes. The study included 226 adolescents and their primary caregivers; they were then randomized into a coping skills group or an education group. This review conducted phone interviews with 13 of those patient-parent duos. The first part of the interview discussed the patient experiences and the second half discussed what the participants would like to be included in further programs.

All parents and most of the adolescents in the coping skills group felt their problem-solving strategies improved they could more openly discuss the illness and any concerns. While participants from both groups felt the TeamWork project was beneficial, they also felt they began to forget some of the "study strategies" that they were given over time. "Despite the benefits that participants attributed to TeamWork, some coping skills group participants acknowledged that it is easy to forget new T1D skills and strategies over time and therefore it is difficult to enact long-term behavior change" [3]. Most of the parents enjoyed being able to attend the sessions with their child but not all of the adolescents wanted their parents in the session with them. Most adolescents mentioned they would like further sessions on physical activity, nutrition, blood glucose management, stress, school, social support, driving, and post-high school plans. Some parents mentioned they would be interested in regularly scheduled check-ins with psychology. As changing long-term behavior can be difficult it may have been beneficial to provide educational materials and follow-up phone calls to keep them on track.

Markowitz et al. [12] conducted a retrospective study of electronic health records to assess care utilization and glycemic control. The population reviewed included patients with diabetes, $99 \%$ with type-1 diabetes, aged $<30$ with duration of diagnosis $>1$ year. A total of 1771 electron health records from the Joslin Diabetes Center were reviewed based on appointment status and glycemic control. The Joslin Center recommends visits every 3-4 months with a medical provider and to see a registered dietician once per year. Mental health visits are scheduled as needed, the mental health providers include social workers, psychologists and psychiatrists.

Patients in the highest A1c category ( $>9 \%)$ tended to be older with a longer duration of diabetes $(\mathrm{p}=.0001)$, more mental health visits $(\mathrm{p}=$.
0001) and more incomplete visits than those in the lower value A1c groups. As for parental attendance, about $48 \%$ of non-mental health visits were attended by mothers, with fathers alone attending about $11 \%$ of the visits. It was found that parental attendance decreased as the duration of diabetes increased. In the patients whose fathers attended $>1$ visit there was lower A1c values $(\mathrm{p}=.0001)$. Of the $22 \%$ of patients with scheduled mental health appointments, only $84 \%$ completed their appointments. Adolescents accounted for $23 \%$ of the mental health appointments. When compared with to those without mental health visits it was found that patients with one or more mental health visits had higher mean Alc values ( $\mathrm{p}=.0001)$.

The review indicates parental involvement can affect glycemic control, especially when fathers or both parents are involved in the appointment. Markowitz et al. suggest involving an ambassador program to help outreach patients to improve appointment attendance, especially in adolescents as gaps in care are more likely to occur as they age and transfer to adult diabetic providers. Implementing text message reminders and internet-based portals may assist to lower noshow rates especially in the younger populations. "These studies also should examine the impact of mental health interventions on preventing the recognized deterioration in glycemic control that accompanies the older school age and teen years" [6].

In a six-year longitudinal observational study by Hood, et al. [3] psychosocial burden among adolescents was evaluated and was compared to glycemic control for the first six years of diabetes. Data was obtained from SEARCH for Diabetes in Youth, which consists of U.S children diagnosed with diabetes before the age of 20. Data was collected at study visits at their baseline, 12 months, 24 months, and 60 months after baseline. Blood was drawn for glycemic control, demographic information was collected and psychosocial burden was determined using standardized depression and quality of life scales. The Center for Epidemiologic Studies Depression (CES-D) a 20-item self-report questionnaire was administered; higher scores correlate to more depressive symptoms. The Pediatric Quality of Life Inventory (PedsQL) was used; scores range from 0-100 and higher scores indicate better levels of functioning.

The data suggested the first six years of diabetes are plagued with deterioration of glycemic control which could be predicted by sociodemographic variables and changing psychosocial burden. Five variables predicted Alc values at the 60 month follow-up, $(\mathrm{p}<.05)$, these were; longer duration of diabetes, being black/African American or Hispanic, declining diabetes quality of life, and increased generic quality of life. When quality of life scores declined the A1c values began climbing suggesting a link between the two. However, there was not one specific time frame that appeared to affect quality of life scores rather it seemed to stay stable over time. This supports the need for more intervention at the time of diagnosis to help preserve quality of life and work toward establishing coping and problem-solving skills. Although the study looked at both type- 1 and 2 diabetes it did address the youth population and an extremely important topic of psychosocial burden, which is a huge battle when helping young patients deal with their illness.

\section{Results}

The articles reviewed identified associations between mental health issues, such as depression, anxiety, or eating disorders, and poor glycemic control and diabetes self-management. Claude et al. [8] reported health anxiety was present in $1 / 4$ of their respective study 
participants but was more common among younger participants. The implementation of a counseling service improved self-perceived anxiety as well as glycemic control in type-1 diabetics [7], the quality of life improved after mindfulness-based cognitive therapy session [9] and in a 2012 study by Bernstein et al. [2] more than one-third of patients screened positive for anxiety, depression or disordered eating. Patients with positive screens were twice as likely to have poor glycemic control of their diabetes. The results stress the importance of not only screening adolescent type-1 diabetics for mental health issues but also following through with treatment and long-term management. Disordered eating is another major concern for the adolescent type-1 diabetic population [13]. The Diabetes Eating Problem Survey is directed towards adults rather than the pediatric or adolescent population. Adults and adolescents have different concerns in life as well as different learning levels, creating a need for an adolescentspecific questionnaire. In 2010 Markowtiz et al. sought to develop a scale to determine disordered eating among adolescents, the revised DEPS scale produced encouraging validity with the comparison of areas that would be influenced by current disordered eating. They found encouraging validity with the youth population the revised survey was tested on. The results of the revised DEPS were encouraging, Powers and colleagues in 2013 determined used of the DEPS scale may introduce unhealthy eating behaviors based on the questions asked. For example one question on the survey is in regards to insulin manipulation and/or omission for weight control. Powers et al., mention concern for this question as it may influence adoption of unhealthy behaviors that the patient may have previously been unaware of. While many tools are available for screening for eating disorders there are none that specifically address an adolescent type-1 diabetes subpopulation. Bernstein et al. [2] discuss the importance of primary care and subspecialty providers caring for adolescents with type- 1 diabetes being aware of the prevalence of mental health disorders and screening with a referral if necessary to mental health providers. There are many programs for medical management of type-1 diabetes, or for patients with an eating disorder alone, but managing the two together brings many obstacles such as finding mutually agreed upon meal plans and making sure the correct insulin doses are being used. Management of comorbidities requires understanding of both illnesses and an interdisciplinary approach with multiple providers being aware of treatment plans [1].

Parental influence was noted to play an important role in the glycemic control of the type-1 diabetic population. Hebert et al 2013 reported parents and adolescents attending education sessions, both together and separately, felt more confident and comfortable in discussing the illness management with each other. In a separate study by Markowitz et al. [6] parental presence, especially fathers influenced and improved glycemic control. Patients who had scheduled mental health visits tended to have higher A1c values, an indication for early identification of mental health issues to prevent deterioration of glycemic control.

\section{Discussion}

The integrative review focused on mental health issues in adolescent patients with type-1 diabetes and revealed three major illnesses seen including depression, anxiety and disordered eating. Of the articles reviewed patients with mental health were noted to experience more negative effects with glycemic control and diabetes self-management. In 2014 Hood et al. [4], reported adolescent patients with decreased diabetes specific quality of life scores tended to have higher Alc values.
Bernstein et al. [1], also reported adolescents with type-1 diabetes that screened positive for anxiety, depression and disordered eating has worsened glycemic control than those adolescents without a mental health disorder.

McGrady and Hood conducted a study in 2010 utilizing the Children's Depression Inventory (CDI) with a high internal consistency (Cronbach's $\alpha=0.84$ ), to assess for depression among adolescents with type- 1 diabetes. The results revealed a decrease in blood glucose monitoring and an increase in Alc values in the adolescents that reported increased depressive symptoms such as negative mood and ineffectiveness. The use of a self-reporting questionnaire rather than a clinical assessment was a limitation to the McGrady and Hood, 2010 study. In addition, changes in depressive symptoms were not addressed over time.

Markowitz et al. utilized a disordered eating screening tool for adults with diabetes to create a new screen tool focused toward an adolescent population. The questionnaire at hand was the Diabetes Eating Problem Survey and was originally created for an adult type- 1 diabetic population to screen for disordered eating. The current survey has a strong internal consistency (Cronbach's $\alpha=0.95$ ) and was strongly correlated to diabetes specific stressors $(\mathrm{p}<0.001)$. The questionnaire was then revised to exclude duplicate questions and those that did not measure disordered eating. The revised 16-question DEPS-R can be used in an adolescent type-1 diabetic population to screen for disordered eating. The DEPS-R was found to have a strong internal consistency with the sample of youth with type-1 diabetes (Cronbach $\alpha=0.86$ ). Overall the use of the DEPS-R showed promising external consistency and should be considered as a screening tool for the adolescent type- 1 diabetic population. The small size and narrow agerange posed limitations and generalizability of the results. However the DEPS-R was not validated against an established disordered eating screening tool.

In 2014 Janzen Claude and colleagues studied the prevalence and implications of health anxiety in a diabetic population. They utilized the Health Anxiety Inventory-Short Form (SHAI) with an internal consistency of Cronbach's $\alpha=.88$, the State-Trait Anxiety Inventory (STAI), with an internal consistency of Cronbach's $\alpha=.94$, the Fear of Complications Questionnaire, with internal consistency of Cronbach's $\alpha=.92$, the World Health Organization Quality of Life BREF (WHOQOL-BREF) with internal consistency of Cronbach's $\alpha=.84$. Lastly the Summary of Diabetes Self-Care Activities (SDSCA) scale was used, only sections of it were used as not all applied to the population. The four subscales used were dietary $\alpha=.67$, exercise $\alpha=.89$, blood glucose testing $\alpha=.83$ and medication $\alpha=.83$. Higher anxiety levels were found among younger patients, female, single and those with a shorter time since diagnosis. Each screening tool utilized offered good internal consistency and helped identify those patients experiencing health anxiety. The study relied on self-report rather than clinical evaluation, which may not provide completely accurate results. Some patients may tend to overestimate blood glucose monitoring, whether they are unaware of how often they actually monitor or they may be trying to appear to be more compliant than they are. The study also utilized volunteers rather than a randomly selected group, volunteers may already have health anxiety and are therefore more willing to participate. A random sample may be more beneficial to accurately represent the population, correlational findings were used but longitudinal findings would provide greater benefit.

Hood et al., conducted a study in 2014 using $\mathrm{n}=1307$ adolescent patients with both type- 1 and type- 2 diabetes. Two scales were used 
over several point periods to assess for longitudinal outcomes, they included the Center for Epidemiologic Studies Depression scale (CESD), and the Pediatric Quality of Life Inventory (PedsQL). The results revealed decreasing quality of life scores over time and were predictors of increased A1c values. Four variables found to predict higher A1c values were younger age, longer duration of diabetes, being Black/ African, as well as decreasing diabetes quality of life over time. Similarly, Janzen Claude et al., reported a younger age was linked to higher levels of anxiety in the diabetic population, but they also found that a shorter time from diagnosis was likely to cause increased anxiety levels. A limitation of this study was that visits to evaluate psychosocial burden were at 1, 2 and 5 years. The study design did not determine if any significant changes occurred between the visits, increased frequency of visits and evaluation may have been more beneficial, making it easier to detect subtle changes. Measuring Alc values was the only method used of determining diabetic control, and they did not assess for hypo or hyperglycemic episodes.

Bernstein et al., [2] utilized three validated mental health disorder screening tools including, Becks Depression Inventory, the Screen for Child Anxiety Related Emotion Disorders, and the Eating Disorder Screen for Primary Care. Nearly one-third of participants screened positive on at least one instrument. The participants most likely to have poor diabetes control were younger participants and those with a longer duration of diabetes. Both of these findings correlate with the 2014 study conducted by Hood et al., which also found increased levels of anxiety among younger participants and those diagnosed with diabetes at a younger age. Janzen Claude et al. also found higher anxiety in younger and female patients. Limitations of this study are the participant population was taken from a diabetes center with a high prevalence of Latinos; the surveys were also administered in English, which may have not been fully understood by all participants. Parents were also sitting close by the children as they completed the screening tools, which may have influenced their answers.

In summary most studies utilized screening tools depend on selfreport, these reports revealed mental health disorders including depression, anxiety and disordered eating were not uncommon among the populations questioned. A common thread among the studies was an increased prevalence of mental health issues in the younger populations. This helps validate the need for increased screening and more specific screening tools for the adolescent type-1 diabetic population. Majority of the articles looked to validate screening tools or simply to assess for mental health disorders among a population. A lack of follow through was noticed. Identifying an issue is an important step but actually following up and treating an issue is just as important.

\section{Implications for Practice}

Many of the studies reviewed utilized screening tools to assess for mental health issues and worked toward creating a validated screening tool. The studies found positive results and correlations between mental health issues and decreased glycemic control. Identifying the issue is of high importance on the primary care setting. As nurse practitioners it will be important to identify an appropriate time to have the patient complete the chosen questionnaire and review it prior to the end of the appointment. As Bernstein et al., [2] pointed out, having a parent sitting next to the adolescent while they complete the questionnaire may affect the outcome. Therefore, parental permission should be obtained to bring the patient to a safe and comfortable place where they can honestly complete the survey without any interference. The primary care provider must also be cautious of what surveys they are using and the population they are using them with. Some surveys may teach disordered eating behaviors that the patient may not have previously been aware of. Powers et al., identified a limitation to the DSED-M and DEPS surveys as their questions regarding insulin omission may lead to patient's adopting unhealthy behaviors that they may not have previously been aware of. All surveys completed must be discussed with the patient to determine how they came about answering the positive questions and to further explore their feelings. Once a patient has been identified with a mental health issue it must be appropriately addressed, including a mental health referral, or having on-site counselors would provide adequate follow up to help treat the disorder before it becomes severe and dangerous to the patient's overall health. Masding et al. found an overall decrease in A1c values after implementing a six-week counseling service. Patients should be referred to a mental health counselor for further evaluation and treatment of the mental health disorder.

\section{Future Research}

The creation of one unified screening tool for use within an adolescent type-1 diabetic population would be immensely useful, making it easier for providers to implement this way they would not have to choose between multiple screening tools. There are multiple questionnaires to assess for depression, quality of life, disordered eating and anxiety. It is not realistic to hand over a pile of questionnaires and ask the patient to complete them prior to the appointment. Further research should focus on creating one questionnaire to address the domains of mental health (anxiety, depression, disordered eating, quality of life etc.) for the adolescent type-1 diabetic population to complete. Further research on treatment of these disorders would be beneficial. Treatment of disordered eating will be different with the type-1 diabetic patient as there are already nutritional restrictions and guidelines that need to be followed. There should be more randomized controlled trials (level II evidence) look at group or individual counseling and the long-term outcomes on glycemic control and overall quality of life. A meta-analysis of multiple randomized controlled trials will provide a high level of evidence and a solid research base to implement changes with.

\section{Conclusion}

In conclusion, the integrative review worked to illustrate the vast presence of mental health issues in adolescents with type-1 diabetes. Negative quality of life and poor disease management was seen with these patients. Although there are screening tools available it would be beneficial to have a more focused on for this specific population. There were counseling sessions and mindfulness-based cognitive therapy that showed promising outcomes to help decrease some of the mental health issues seen including anxiety and depression. This integrative review brought up some very important aspects of caring for adolescent type- 1 diabetics and helped to reveal areas that still need further work and provider education.

\section{References}

1. American Diabetes Association (2014) Type 1 Diabetes.

2. Bernstein CM, Stockwell MS, Gallagher MP, Rosenthal SL, Soren K (2013) Mental health issues in adolescents and young adults with type 1 diabetes: prevalence and impact on glycemic control. Clin Pediatr (Phila) 52: $10-15$.

3. Hood KK, Beavers DP, Yi-Frazier J, Bell R, Dabelea D, et al. (2014) Psychosocial burden and glycemic control during the first 6 years of 
Citation: Goad K (2015) Examining the Mental Health Issues Prevalent in Adolescent Type-1 Diabetics: An Evidence-Based Review to Improve Health. J Diabetes Metab 6: 599. doi:10.4172/2155-6156.1000599

Page 7 of 7

diabetes: results from the SEARCH for Diabetes in Youth study. J Adolesc Health 55: 498-504.

4. McGrady ME, Hood KK (2010) Depressive symptoms in adolescents with type 1 diabetes: associations with longitudinal outcomes. Diabetes Res Clin Pract 88: e35-37.

5. Melnyk B, Fineout-Overholt E (2011) Evidence-based practice in nursing \& healthcare: A guide to best practice (2nd edtn). Wolters Kluwer/ Lippincott Williams \& Wilkins, Philadelphia.

6. Markowitz JT, Butler DA, Volkening LK, Antisdel JE, Anderson BJ, et al. (2010) Brief screening tool for disordered eating in diabetes: internal consistency and external validity in a contemporary sample of pediatric patients with type 1 diabetes. Diabetes Care 33: 495-500.

7. Masding M, Ashley K, Klejdys S (2011) Introduction of a counselling service for patients with type 1 diabetes: better glycaemic control and reduced anxiety. Practical Diabetes International, 28: 28-30.

8. Janzen Claude JA, Hadjistavropoulos HD, Friesen L (2014) Exploration of health anxiety among individuals with diabetes: prevalence and implications. J Health Psychol 19: 312-322.
9. van Son J, Nyklícek I, Pop VJ, Blonk MC, Erdtsieck RJ, et al. (2013) The effects of a mindfulness-based intervention on emotional distress, quality of life, and $\mathrm{HbA}(1 \mathrm{c})$ in outpatients with diabetes (DiaMind): a randomized controlled trial. Diabetes Care 36: 823-830.

10. Powers MA, Richter S, Ackard D, Critchley S, Meier M, et al. (2013) Determining the influence of type 1 diabetes on two common eating disorder questionnaires. Diabetes Educ 39: 387-396.

11. Herbert LJ, Sweenie R, Kelly KP, Holmes C, Streisand R (2014) Using qualitative methods to evaluate a family behavioral intervention for type 1 diabetes. J Pediatr Health Care 28: 376-385.

12. Markowitz JT, Volkening LK, Laffel LM (2014) Care utilization in a pediatric diabetes clinic: cancellations, parental attendance, and mental health appointments. J Pediatr 164: 1384-1389.

13. Joslin Diabetes Center (2015) Young Adult Transition Care Program for Type 1 and Type 2 Diabetes. 